\title{
Characterizing the radio continuum emission from intense starburst galaxies
}

\author{
T. J. Galvin,,${ }^{1,2,3 \star}$ N. Seymour, ${ }^{2}$ M. D. Filipović, ${ }^{1}$ N. F. H. Tothill, ${ }^{1}$ J. Marvil, ${ }^{3}$ \\ G. Drouart, ${ }^{2}$ M. Symeonidis ${ }^{4}$ and M. T. Huynh ${ }^{5}$ \\ ${ }^{1}$ Western Sydney University, Locked Bag 1797, Penrith, NSW 2751, Australia \\ ${ }^{2}$ International Centre for Radio Astronomy Research, Curtin University, Perth, WA, Australia \\ ${ }^{3}$ CSIRO Astronomy and Space Science, PO Box 76, Epping, NSW 1710, Australia \\ ${ }^{4}$ Mullard Space Science Laboratory, University College London, Holmbury St Mary, Dorking, Surrey RH5 6NT, UK \\ ${ }^{5}$ International Centre for Radio Astronomy Research, M468, University of Western Australia, Crawley, WA 6009, Australia
}

Accepted 2016 May 26. Received 2016 May 11; in original form 2015 December 14

\begin{abstract}
The intrinsic thermal (free-free) and non-thermal (synchrotron) emission components that comprise the radio continuum of galaxies represent unique, dust-free measures of star formation rates (SFR). Such high SFR galaxies will dominate the deepest current and future radio surveys. We disentangle the thermal and non-thermal emission components of the radio continuum of six ultraluminous infrared galaxies $\left(L_{\mathrm{FIR}}>10^{12.5} \mathrm{~L} \odot\right)$ at redshifts of $0.2 \leq z \leq 0.5$ and 22 IR selected galaxies. Radio data over a wide frequency range $(0.8<v<10 \mathrm{GHz})$ are fitted with a star-forming galaxy model comprising of thermal and non-thermal components. The luminosities of both radio continuum components are strongly correlated to the $60 \mu \mathrm{m}$ luminosity across many orders of magnitude (consistent with the far-IR to radio correlation). We demonstrate that the spectral index of the radio continuum spectral energy distribution is a useful proxy for the thermal fraction. We also find that there is an increase in mean and scatter of the thermal fraction with FIR to radio luminosity ratio which could be influenced by different time-scales of the thermal and non-thermal emission mechanisms.
\end{abstract}

Key words: galaxies: starburst - radio continuum: galaxies.

\section{INTRODUCTION}

The far-IR to radio correlation (FRC) is an observed tight linear relationship between the radio and infrared luminosities of local star-forming galaxies (SFGs). Introduced by van der Kruit (1971), van der Kruit (1973) and subsequently studied by many authors (e.g. de Jong et al. 1985; Helou, Soifer \& Rowan-Robinson 1985; Condon 1992; Yun, Reddy \& Condon 2001) the correlation holds across many orders of magnitude and is thought to be due to both luminosities tracing star formation rates (SFRs).

Simple models devised by Voelk (1989) and Lisenfeld, Voelk \& Xu (1996) describe a process whereby dust is heated by ultraviolet (UV) and optical radiation emitted by a combination of high-mass stars $\left(M>8 \mathrm{M}_{\odot}\right)$, associated with recent star formation and contributing to the bulk of emission, and a population of older lower mass stars. Energy reradiated from the dust produces the total far-infrared (FIR) emissions of a galaxy. $\mathrm{H}$ II regions surrounding these high-mass stars produce thermal emission in the radio domain originating from free-free interactions of ionized particles.
After $\sim 10$ Myr these high-mass stars go supernova and produce non-thermal radio emission from ultrarelativistic electrons interacting with large-scale galactic magnetic fields. The FIR and radio continuum emission become correlated with scatter likely due to age effects.

The derived radio luminosity to SFR relationship relies heavily on the FRC (Kennicutt 1998; Bell 2003). In order to use the FRC as a method to measure SFRs in the distant Universe, it is important to investigate this correlation at higher luminosities, a regime typically made up of high-redshift galaxies. In the local Universe, Hughes et al. (2006) and Murphy et al. (2006) have shown cases where deviations occur from the correlation within galaxies. However, it is thought that this scatter is brought about by a longer mean free path of the relativistic electrons compared to the dust heating UV photons and does not necessarily represent its break down. Appleton et al. (2004) and Garrett (2002) have shown that at redshift $z \leq 1$ the correlation (mostly) is correct. Although subsequent millimetre and submillimetre observations by Kovács et al. (2006) and Vlahakis, Eales \& Dunne (2007) show that at high redshift $(z \geq 1)$ there is a decrease of $\sim 0.2$ dex in the correlation ratios when compared to local samples, Seymour, Dwelly \& et al. (2008) demonstrate that by removing active galactic nucleus (AGN) contamination and 
comparing local and distant starburst galaxies the relationship remains intact.

Seymour et al. (2011) used infrared data from the Herschel Space Observatory and Spitzer Space Telescope to estimate total $(8-1000 \mu \mathrm{m})$ infrared luminosities for strong radio sources $(25 \leq$ $\left.\log \left(L_{1.4} / \mathrm{W} \mathrm{Hz}^{-1}\right) \leq 26.5\right)$ in the extragalactic Spitzer First Look Survey field. These sources, ranging from moderate to high redshift $(0.4<z<3.0)$, show a continued strong correlation between the radio and FIR luminosities. Similarly, Ivison et al. (2010) used flux-limited $250 \mu \mathrm{m}$ and $1.4 \mathrm{GHz}$ samples from Herschel and the Very Large Array (VLA), respectively, in order to investigate how the ratio of these components may vary with respect to redshift, luminosity and temperature. Again, their results show a continued correlation between the FIR and radio luminosities at $z<2$.

Modelling work by Bressan, Silva \& Granato (2002) and Ivison et al. (2010) shows that the FRC is a natural product of any dusty starburst galaxy where synchrotron emission dominates over inverse-Compton emission, and the fading rate of supernovae is longer than the electron cooling time. Deviations from this natural correlation are thought to be possible, particularly in the cases where radio thermal emission dominates over the radio non-thermal emission, such as in the early phase of a starburst. Additionally, the correlation ratios may be affected depending on the size of volume over which the star formation occurs (Clemens et al. 2008).

Along these lines, Murphy (2009) makes predictions on how the behaviour of the FRC may change as a function of redshift due to changing intergalactic medium conditions. Primarily due to increasing inverse-Compton losses to cosmic ray electrons from the cosmic microwave background, the non-thermal emission of SFGs will become increasingly suppressed at higher redshifts.

Radio continuum surveys use, almost exclusively, the $1.4 \mathrm{GHz}$ monochromatic luminosity of a galaxy to estimate SFRs. At this frequency the spectrum is composed predominantly of non-thermal synchrotron radiation caused by ultrarelativistic electrons accelerated by the shocks of supernovae of massive stars $\left(M>8 \mathrm{M}_{\odot}\right)$ and may be used as a non-instantaneous tracer of star formation. However, a better tracer would be the thermal free-free emission originating from $\mathrm{H}_{\mathrm{II}}$ regions surrounding high-mass stars. Not only is this type of emission a direct tracer of current SFRs of high-mass stars, but it also becomes the dominant emission mechanism at $v \geq 10 \mathrm{GHz}$. This has the advantage of being capable of probing higher redshifts (Murphy et al. 2014). It is expected that the deep extragalactic radio surveys produced by the next generation of radio telescopes (e.g. ASKAP, MeerKAT, JVLA, LOFAR, eMERLIN and SKA) will be composed predominantly of powerful SFGs at $z \geq 1$ with radio luminosities consistent with very high formation rates $\left(\geq 100 \mathrm{M}_{\odot} \mathrm{yr}^{-1}\right)$.

In order for radio observations to be a useful tracer for the SFR of distant, high-redshift galaxies, it is first necessary to understand how the radio continuum components themselves may be related to the FIR of the galaxy and how their relatively strengths can be measured. Ultraluminous infrared galaxies (ULIRGs; $L_{\mathrm{IR}}^{8-1000 \mu \mathrm{m}}>$ $10^{12} \mathrm{~L}_{\odot}$ ) represent the class of sources most analogous to those anticipated in future radio continuum surveys. Price \& Duric (1992) studied 31 galaxies in the local Universe $(z<0.02)$ and have shown that not only are the non-thermal and thermal components able to be separated, but they can form their own correlations with FIR luminosities. Understanding how these components evolve with increasing redshift will be required so that the FRC can continue to be used as a measure of SFR at high redshifts.

In this study, we investigate the FRC in a set of distant ULIRGs at redshifts between 0.2 and 0.5 . By constructing spectral energy distributions (SEDs) across multiple frequency domains we further investigate the astrophysics of the FRC. We assume a flat Universe, where $\Omega_{\mathrm{m}}=0.3, \Omega_{\lambda}=0.7$ and $H_{0}=70 \mathrm{~km} \mathrm{~s}^{-1} \mathrm{Mpc}^{-1}$.

\section{STAR-FORMING SAMPLE AND DATA REDUCTION}

\subsection{Sample selection}

ULIRGs were targeted in this study as their high FIR emission indicates strong star formation, making them useful analogues of higher redshift galaxies which dominate deep radio surveys. We choose a redshift range beyond the local Universe, $z>0.1$, as local ULIRGs are rare and not always representative of distant ULIRGs.

Our sample was taken from Braun et al. (2011), who observed the $\mathrm{CO}(1-0)$ transition in a set of 11 ULIRGs. Braun et al. identified these sources from the NASA/IPAC Extragalactic Database (NED) ${ }^{1}$ using the following criteria:

(i) FIR detections at 60 and $100 \mu \mathrm{m}$;

(ii) spectroscopic redshifts between 0.2 and 0.5 ;

(iii) FIR luminosities $(40-500 \mu \mathrm{m}) L_{\mathrm{FIR}}>10^{12.5} \mathrm{~L}_{\odot}$;

(iv) declinations south of $-12^{\circ}$.

The sample used here is based on that from Braun et al. (2011) who considered all objects matching these selection criteria known by NED at the time that the project was planned. We also include IRAS F00183-7111 as it has been extensively studied in the literature (Spoon et al. 2009; Norris et al. 2012; Mao et al. 2014, and references within). Referring to the Revised IRAS Faint Source Redshift Catalog (RIFSCz; Wang et al. 2014) we note an additional seven sources matching these criteria, and a further 12 with photometric redshifts that lie within this redshift range. To assess whether our sample was representative of all available sources a series of two sided Kolmogorov-Smirnov tests were performed on key intrinsic parameters listed in the RIFSCz. The $p$-values returned in these tests show no evidence to suggest that there is a different underlying distribution for most properties, including dust mass, as well as proxies for stellar mass and extinction. The FIR luminosities and derived SFRs of the sources matching these criteria, but not included here, are marginally lower $(\sim 0.1 \mathrm{dex})$ on average. However, this is not a significant bias as the goal of this project is to observe the most highly SFGs.

\subsection{Observational data}

\subsubsection{Radio continuum}

The 12 ULIRGs were observed using the Australian Telescope Compact Array (ATCA; Wilson et al. 2011), under project C2696 (PI: N. Seymour), for a $20 \mathrm{~h}$ period during 2012 August, using the $2.048 \mathrm{GHz}$ spectral window provided by the Compact Array Broadband Backend (CABB) filters. $6 \mathrm{GHz}$ of spectral information was collected around three central frequencies $(v=2.1,5.5$ and $9.0 \mathrm{GHz}$ ). The sparsely distributed $6 \mathrm{~A}$ array configuration was used to isolate sources in the sky. At $v=2.1 \mathrm{GHz}$ this array configuration gives a restoring beam of roughly 7 arcsec, though this can vary depending on the $u v$ sampling of our sources or the numbers of subbands imaged (see Section 2.3). This size was sufficient to prevent confusion effects and, based on extracted source sizes for

\footnotetext{
${ }^{1}$ http://ned.ipac.caltech.edu/
} 
Table 1. A summary of the sources observed in the ATCA project C2696 and used in this study. The FIR luminosities have been taken from Spoon et al. (2009) and Braun et al. (2011).

\begin{tabular}{lcccc}
\hline $\begin{array}{l}\text { Source name } \\
\text { IRAS }\end{array}$ & $\begin{array}{c}\text { RA (J2000) } \\
(\mathrm{h} \mathrm{m} \mathrm{s})\end{array}$ & $\begin{array}{c}\text { Dec. }(\mathrm{J} 2000) \\
\left({ }^{\prime}{ }^{\prime \prime}\right)\end{array}$ & $z$ & $\begin{array}{c}L_{\mathrm{FIR}} \\
\log \left(\mathrm{L}_{\odot}\right)\end{array}$ \\
\hline F00183-7111 & $00: 20: 34.7$ & $-70: 55: 27$ & 0.327 & 12.95 \\
F00320-3307 & $00: 34: 28.5$ & $-32: 51: 13$ & 0.439 & 12.68 \\
$00397-1312$ & $00: 42: 15.5$ & $-12: 56: 03$ & 0.262 & 12.67 \\
$00406-3127$ & $00: 43: 03.2$ & $-31: 10: 49$ & 0.342 & 12.58 \\
$02262-4110$ & $02: 28: 15.2$ & $-40: 57: 16$ & 0.493 & 12.50 \\
$02456-2220$ & $02: 47: 51.3$ & $-22: 07: 38$ & 0.296 & 12.58 \\
$03538-6432$ & $03: 54: 25.2$ & $-64: 23: 45$ & 0.301 & 12.62 \\
F04565-2615 & $04: 58: 34.7$ & $-26: 11: 14$ & 0.490 & 12.78 \\
$07380-2342$ & $07: 40: 09.8$ & $-23: 49: 58$ & 0.292 & 12.54 \\
$23515-2917$ & $23: 54: 06.5$ & $-29: 01: 00$ & 0.335 & 12.52 \\
F23529-2119 & $23: 55: 33.0$ & $-21: 03: 09$ & 0.429 & 12.67 \\
F23555-3436 & $23: 58: 06.5$ & $-34: 19: 47$ & 0.490 & 12.63 \\
\hline
\end{tabular}

our sample from the SuperCosmos project (Hambly et al. 2001), avoids resolving out extended source structure.

Each source was observed for approximately $11 \mathrm{~min}$ at $2.1 \mathrm{GHz}$ and $45 \mathrm{~min}$ at 5.5 and $9.0 \mathrm{GHz}$. Sources were observed at least five times at each frequency throughout the observing run. The primary calibrator PKS 1934-638 was used to provide flux density and bandpass calibration solutions across all central frequencies. A total of eight phase calibrators were used due to the spatial distribution of the program sources.

\subsubsection{Infrared}

The 60 and $100 \mu \mathrm{m}$ infrared flux densities of the sample sources were obtained from the IRAS source catalogues (Moshir 1990). A summary of these measurements is shown in Table 1. The FIR luminosities have been taken from Spoon et al. (2009) and Braun et al. (2011).

\subsubsection{Other}

NED was used to collect additional flux density measurements for each source. We searched for broad-band flux density measurements in the ranges $0.1<v<100 \mathrm{GHz}$ and $37<\lambda<500 \mu \mathrm{m}$ (Table A1).

The listed NRAO VLA Sky Survey (NVSS) detection of IRAS F00320-3307 at $1.4 \mathrm{GHz}$ was $\sim 2.6$ times higher than our $2.1 \mathrm{GHz}$ ATCA measurement, and therefore not included in its modelling.

\subsection{Radio continuum data reduction}

The MIRIAD (Sault, Teuben \& Wright 2011) and KARMA (Gooch 2011) software packages were used for data reduction and analysis. The guided automated flagging MIRIAD routine PGFLAG was used in conjunction with more traditional MIRIAD flagging and calibration tasks in order to perform an initial data reduction. Given the large fractional bandwidth provided by CABB, MFCLEAN (Sault \& Wieringa 1994) was used to deconvolve the multifrequency synthesized dirty map.

Once a calibration solution was applied to each of the observations program sources, they were then imaged individually across all frequency bands $(v=2.1,5.5$ and $9.0 \mathrm{GHz})$ using their complete bandwidth $(\Delta v=2.048 \mathrm{GHz}$, minus the edge channels automatically flagged by ATLOD). A Briggs robust parameter corresponding

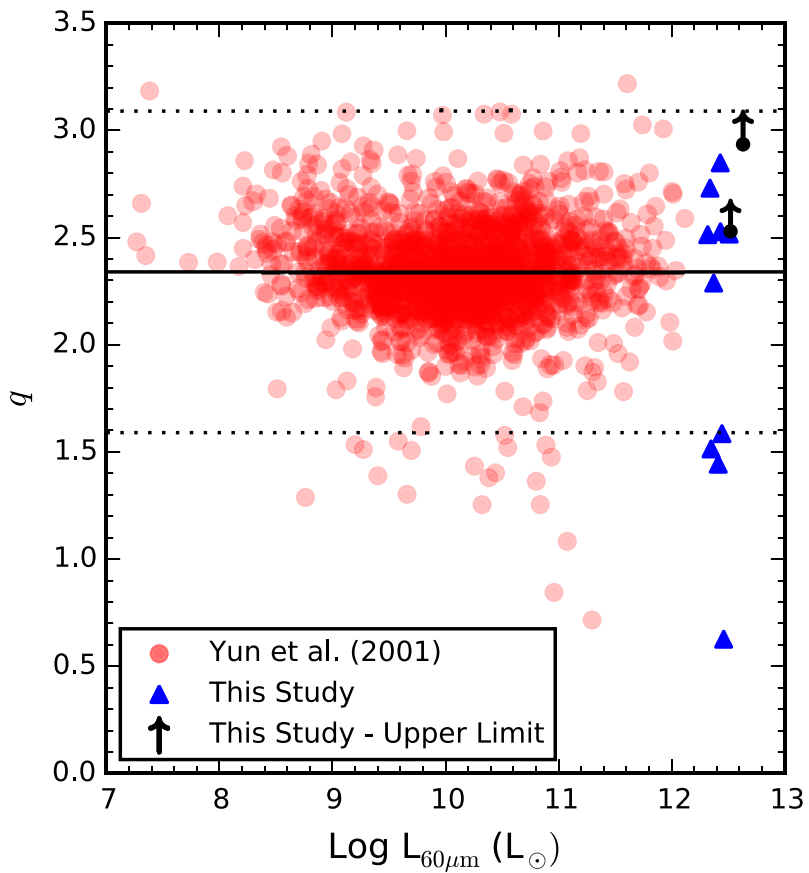

Figure 1. A comparison between the $60 \mu \mathrm{m}$ luminosity and the derived $q$ of sources from Yun et al. (2001) and this study. The solid horizontal line represents the mean $q=2.34$, as calculated by Yun et al. (2001). The dotted lines represent the radio-excess (below) and IR-excess (above) objects, defined as three times the standard deviation (SD) of $q(\mathrm{SD}=0.25)$ from the Yun et al. (2001) sample.

to natural weighting was used to provide the maximum signalto-noise ratio (SNR). These preliminary images were produced in order to compare the calibration among the $u v$-data sets. During this stage it was found that sources IRAS 07380-2342 and IRAS F23555-3436 (marked as black arrows in Fig. 1) were not detected at any frequency. There is a known infrared AGN component associated with IRAS 07380-2342 (Han \& Han 2012, and references therein), boosting its FIR luminosity. The radio luminosity inferred from the FIR luminosity without the AGN component is consistent with our detection limits.

Images were then created with a consistent angular resolution in order to prevent emission from extended structure being resolved out at higher frequencies. This was done by convolving each of the higher frequency images with a Gaussian that matched the resolution of the lowest frequency image. We convolved the image to the desired resolution as the $u v$-coverage of the data is limited and clipping the $u v$-data would have increased the difficulty of the imaging processes of the remaining $u v$-data.

By exploiting the $\sim 2 \mathrm{GHz}$ bandwidth provided by the ATCA CABB system, we were able to produce a more densely sampled SED of most sources. If there was a sufficient SNR in the fitted point source model $(\mathrm{SNR}>8$ ) for a particular image, its corresponding $u v$-file would be split into two half bandwidth files. This $u v$ splitting took into account the flagged channels to ensure that all split $u v$-files had a similar number of useable, unflagged channels. These two new $u v$-files were then re-imaged with natural weighting and their fitted flux values replaced the original. Again, the MIRIAD task CONVOL was used to produce a consistent resolution among image sets.

Point source models were fitted to each source of interest using the MIRIAD task IMFIT in order to estimate their integrated flux density 
and uncertainties. With such an approach, sources with high SNR were split into multiple data points, which could be used to better constrain the radio continuum emission models. A complete list of all convolving beam sizes and flux density measurements is presented in Table A2.

\section{THE FIR TO RADIO CORRELATION}

The FRC has been parametrized by ' $q$ ', defined as the logarithmic ratio between the FIR flux and $1.4 \mathrm{GHz}$ flux density of an object:

$q \equiv \log \left(\frac{\mathrm{FIR}}{3.75 \times 10^{12} \mathrm{~W} \mathrm{~m}^{-2}}\right)-\log \left(\frac{S_{1.4 \mathrm{GHz}}}{\mathrm{W} \mathrm{m}^{-2} \mathrm{~Hz}^{-1}}\right)$,

where $S_{1.4 \mathrm{GHz}}$ is in units of $\mathrm{W} \mathrm{m}^{-2} \mathrm{~Hz}^{-1}$, and FIR is defined as

FIR $\equiv 1.26 \times 10^{-14}\left(2.58 S_{60 \mu \mathrm{m}}+S_{100 \mu \mathrm{m}}\right) \mathrm{W} \mathrm{m}^{-2}$,

where $S_{60 \mu \mathrm{m}}$ and $S_{100 \mu \mathrm{m}}$ are the 60 and $100 \mu \mathrm{m}$ band flux densities from IRAS in Jy (Helou et al. 1985).

Most SFGs have a $q$ scattered around a value of 2.34. IR-excess sources $(q \gtrsim 3)$ may be highly obscured compact starburst galaxies or dust-enshrouded AGN, and some of the dispersion may be influenced in part by variation in extinction and dust temperature. Radio-excess objects $(q \lesssim 1.6)$ are caused by excess radio emission originating from an AGN component in the galaxy (Yun et al. 2001).

It is important to ensure that our sample remains free of radioloud AGN that may otherwise contaminate the results. To help determine the nature of each galaxy included in this study, their $q$ values were evaluated. Initially, we estimate the flux density at $1.4 \mathrm{GHz}$, in the observed frame, using a fitted power law to all radio continuum measurements $\left(S \propto \nu^{\alpha}\right)$. Flux densities at 60 and $100 \mu \mathrm{m}$ from the IRAS faint source catalogue were used to calculate the FIR according to equations (1) and (2). Though there would be a small change in $q$ if it were derived in the rest frame (Seymour et al. 2009), there would be no change to how we have classified the sources in this study.

We compared our sources to those listed by Yun et al. (2001), who produced an infrared flux-limited $\left(S_{60 \mu \mathrm{m}} \geq 2 \mathrm{Jy}\right)$ complete sample of 1809 galaxies by identifying radio counterparts for galaxies in the IRAS Redshift Survey using the NVSS up to a redshift of 0.15. Our sample, however, have redshifts in the range of $0.2<z<0.5$ and target ULIRGs specifically, meaning that the FIR luminosity is often orders of magnitude higher than those seen by Yun et al. (2001). This infrared emission implies a higher SFR for these galaxies, as well as increased radio luminosities.

Of the Yun et al. (2001) sample s $^{2} 98$ percent of sources had $L_{60}$ um luminosities that (once converted back to flux densities) were 2.6 times less than their reported $S_{60 \mu \mathrm{m}}$ fluxes. For this reason, we re-derived the $60 \mu \mathrm{m}$ luminosities of the Yun et al. sample using their reported $60 \mu \mathrm{m}$ flux densities and distances. These re-derived $60 \mu \mathrm{m}$ luminosities are used throughout this paper.

We compared derived $q$ values of sources in Yun et al. (2001) and this study as a function of IRAS $60 \mu \mathrm{m}$ luminosities (Fig. 1). Sources we class as having excess emission are consistent with those used by Yun et al. (2001), who defined it by five times the linear FRC. Sources 03538-6432, F00183-7111, F04565-2615 and F23529-2119 were classed as radio-excess $(q \lesssim 1.6)$, which is indicative of AGN activity, and were thus excluded from subsequent

\footnotetext{
2 The catalogue obtained from the ViZieR (http://vizier.u-strasbg.fr) catalogue service appears to be the source of the inconsistency, as Yun et al. (2001) present the correct luminosities in their study.
}

analysis throughout this study. After removing these four sources, there were eight remaining in our sample, two of which are radio upper limits. We also inspected the IRAS 25 and $60 \mu \mathrm{m}$ flux density ratios and IR template fitting from Wang et al. (2014) of sources in our sample. Of the six sources with radio detections that were not classified as radio-excess, only IRAS $02262-4110$ possessed an elevated $S_{25 \mu \mathrm{m}} / S_{60 \mu \mathrm{m}}$ ratio and included an AGN component in the fitted IR templates. We subtracted the AGN template (determined to be 6 and 2 per cent at 60 and $100 \mu \mathrm{m}$, respectively) from the observed IRAS 60 and $100 \mu \mathrm{m}$ flux densities which were than used throughout this study. The remaining five sources all had $S_{25 \mu \mathrm{m}} / S_{60 \mu \mathrm{m}}$ ratios below 0.3 and were fitted exclusively by starburst-type IR templates. This indicates that there is no detectable AGN component in the 60 and $100 \mu \mathrm{m} I R A S$ flux densities in the remainder of our sample. In terms of the radio continuum however, we are unable to completely dismiss the possibility of weak AGN contamination. Although the $q$ parameter has removed obvious radio-loud AGN sources, it is possible that a low-luminosity AGN may be contributing a portion to the observed radio continuum of sources in our sample. Reviewing the literature sources IRAS 00406-3127, IRAS 02262-4110 and IRAS 23515-2917 have been classified as Seyfert-type galaxies (Allen et al. 1991; Klaas et al. 2001; Véron-Cetty \& Véron 2006). To accurately characterize and correct for any AGN effects that may be present we would need high-resolution radio imaging to resolve the AGN core from extended star formation. Since we do not have these data, we distinguish these three sources 'Seyfert SFG' in subsequent figures to avoid confusion. A brief overview of the literature of the remaining sources in our sample is presented in Appendix B.

\section{SED MODELLING}

We created comprehensive SEDs of each galaxy across the radio and infrared domains using ATCA CABB, IRAS, ISO and VLA data. These SEDs allowed the thermal, non-thermal and dust emissivity components to be modelled and constrained.

\subsection{Emission models}

\subsubsection{Radio continuum}

All galaxy radio SEDs were fitted with a SFG model, which is the sum of thermal and non-thermal emission represented by two distinct power law components (Condon 1992, see fig. 1). This emission model was fitted to all ATCA and VLA flux density measurements in the range from 1.1 to $10 \mathrm{GHz}$ for each source. The emission model is

$S_{v}(v)=A\left(\frac{v}{v_{0}}\right)^{\alpha_{\mathrm{NT}}}+B\left(\frac{v}{v_{0}}\right)^{\alpha_{\mathrm{T}}}$,

where $\alpha_{\mathrm{NT}}$ and $\alpha_{\mathrm{T}}$ are the spectral indices of non-thermal and thermal emission, assumed to be -0.8 and -0.1 , respectively, and $A$ and $B$ represent the fitted normalization parameters. The reference frequency used, $v_{0}$, is $1.4 \mathrm{GHz} /(1+z)$.

\subsubsection{Infrared}

In the infrared domain, a grey body emission model was fitted to each source's SED and was used to describe the dust emissivity of each galaxy. A grey body model is a modified blackbody, where the emissivity is lower than 1 and is scaled with wavelength (Rouan 2011) 
The grey body model (equation 4) was fitted to the IRAS flux density measurements at $\lambda=60$ and $100 \mu \mathrm{m}$, as well as any additional flux density measurements obtained from NED between a wavelength of 37 and $500 \mu \mathrm{m}$ :

$S_{\nu}(\lambda)=N\left[\left(\frac{60 \mu \mathrm{m}}{\lambda}\right)^{3+\beta} \times \frac{1}{\mathrm{e}^{\frac{h c}{\lambda k T}}-1}\right]$,

where $S_{v}$ is the total power radiated in Jy at frequency $v$ (in $\mathrm{Hz}$ ), $T$ is the absolute temperature of the body in $\mathrm{K}, \beta$ is a power with a value between 1 and 2 representing the emissivity and $N$ is a normalization.

Typically, this grey body model contains three free parameters $(N, \beta$ and $T)$. As there were relatively few infrared flux density measurements which could be used, we assign a fixed value of 1.5 to $\beta$. The remaining two parameters ( $T$ and $N$ ) were allowed to vary for all grey body modelling that we performed.

\subsubsection{SED fitting}

A Levenberg-Marquardt least-squares fitting algorithm was used to derive the best-fitting values for all emission models using all available flux density measurements in their observed frame of reference. We estimated the $1.4 \mathrm{GHz}$ and $60 \mu \mathrm{m}$ rest-frame flux densities, after shifting them to the observed frame, using these fitted emission models. The final SEDs produced in this study are presented in Figs 2 and 3 and are composed of all flux density measurements used during the emission modelling (see Tables A1 and A2), the best-fitting SFG and grey body emission models.

\section{RESULTS}

\subsection{SED fits}

In Tables 2 and 3 we present the fitted parameters and their uncertainties (where the uncertainty $\sigma$ for parameter $p$ is defined as $\chi^{2}(p+\sigma)=\chi^{2}(p)+1$; Bevington 1969) which were derived from the fitting procedure. For four of the six sources there were only two infrared flux density measurements available to constrain the grey body emission model, leaving no degrees of freedom. We include in Table 3 both the fitted temperature $(T)$ parameter, derived using the least-squares fitting routine, and the rest-frame temperature $\left(T_{\mathrm{e}}\right)$, where $T_{\mathrm{e}}=T(1+z)$.

In total, six sources were fitted with an SFG emission model (see Table 2). Five of these six sources were dominated by synchrotron non-thermal emission, where the fitted uncertainties ranged between 2 and 30 percent of the corresponding best-fitting value. The fitted thermal components are not as well characterized, since at low frequencies $(v<10 \mathrm{GHz})$ the dominant emission mechanism is typically non-thermal. However we see that for the sixth source, IRAS 23515-2917, there is a strong thermal component with uncertainties similar to those of the best-fitting non-thermal components.

The rest frame dust temperatures (Table 3) for the six SFG type sources are fairly consistent and well constrained. Although we have restricted the parameter space by assigning a constant value to the dust emissivity $\beta$ component, the temperatures agree with similar studies of starburst-type objects with comparable luminosities (Yun \& Carilli 2002; Symeonidis et al. 2013; da Cunha et al. 2015).

\subsection{Comparison to Yun et al. (2001) sample}

The FIR and radio luminosities calculated for sources in this project were compared to the sample compiled by Yun et al. (2001). This comparison (Fig. 4) shows the SFG from this study are in line with the FRC seen by Yun et al. (2001) with small scatter. There is a deficit in the observed $1.4 \mathrm{GHz}$ luminosity of the SFG from this study when compared to the expected $1.4 \mathrm{GHz}$ luminosity based on the FRC. This can be attributed to our object selection method (see Section 6).

Fig. 4 includes the $3 \sigma 1.4 \mathrm{GHz}$ luminosity upper limits of the two sources which had no radio detections (IRAS 07380-2342 and IRAS F23555-3436). These upper limits were derived by scaling the $3 \sigma$ upper limits at $5.5-1.4 \mathrm{GHz}$ using a spectral index of -0.8 $\left(S_{v} \propto v^{-0.8}\right)$ and applying a $k$-correction.

Because we re-derived the $L_{60 \mu \mathrm{m}}$ values from the Yun et al. (2001) catalogue (see Section 3), a new formal fit was calculated. To remain consistent with Yun et al. (2001), no $k$-corrections were applied to sources in their sample. We also excluded AGN or outliers which would disproportionately affect the fitting routine based on their $q$ value and luminosities. Specifically, sources were only included in the fitting process if they satisfied:

(i) $1.64<q<3.04$;

(ii) $6.5<\log L_{60 \mu \mathrm{m}}<13.5$;

(iii) $18.5<\log L_{1.4 \mathrm{GHz}}<25.5$.

Applying these criteria to the original 1809 sources from Yun et al. (2001), a total of 36 were removed. A formal fit to the remaining sources produced an observed radio-FIR luminosity correlation of

$$
\begin{aligned}
\log \left(L_{1.4 \mathrm{GHz}}\right)= & 0.97[ \pm 0.01] \log \left(L_{60 \mu \mathrm{m}} / \mathrm{L}_{\odot}\right) \\
& +12.25[ \pm 0.07] .
\end{aligned}
$$

Although the values of this formal fit are very similar to those of equation (4) of Yun et al. (2001), there is a slight difference in the slope of the FRC, as well as a difference in the radio luminosity normalization component corresponding to $\sim 0.18 \mathrm{dex}$. The fitted uncertainties of each free parameter are identical. We included the normalization uncertainty in Fig. 4.

\subsection{Decomposed radio to FIR}

In order to investigate the thermal and non-thermal radio continuum properties of the Yun et al. (2001) sample, we cross-referenced the 1809 sources in their sample to Dressel \& Condon (1978), who used the Arecibo telescope to observe 2095 galaxies at $v=2380 \mathrm{MHz}$, and the Green Bank $6 \mathrm{~cm}$ radio source catalogue (GB6; Gregory et al. 1996). We were able to identify 22 sources common to all three catalogues using a matching radius of 5 and 20 arcsec, respectively. This provided us with three flux density measurements at $v=1.4$, 2.38 and $4.85 \mathrm{GHz}$ from which we were able to obtain a spectral index and constrain the SFG emission model for these 22 sources.

\subsubsection{Thermal fraction}

As stated by Condon (1992), if integrated flux densities at three or four frequencies are used to fit the (unknown) non-thermal spectral index and the thermal fraction (defined as $A /(A+B)$ from equation 3) simultaneously, the resulting non-thermal spectral index and thermal fraction are strongly correlated (Fig. 5).

This correlation between the thermal fraction and spectral index is expected, as it can be shown numerically that the fitted thermal 

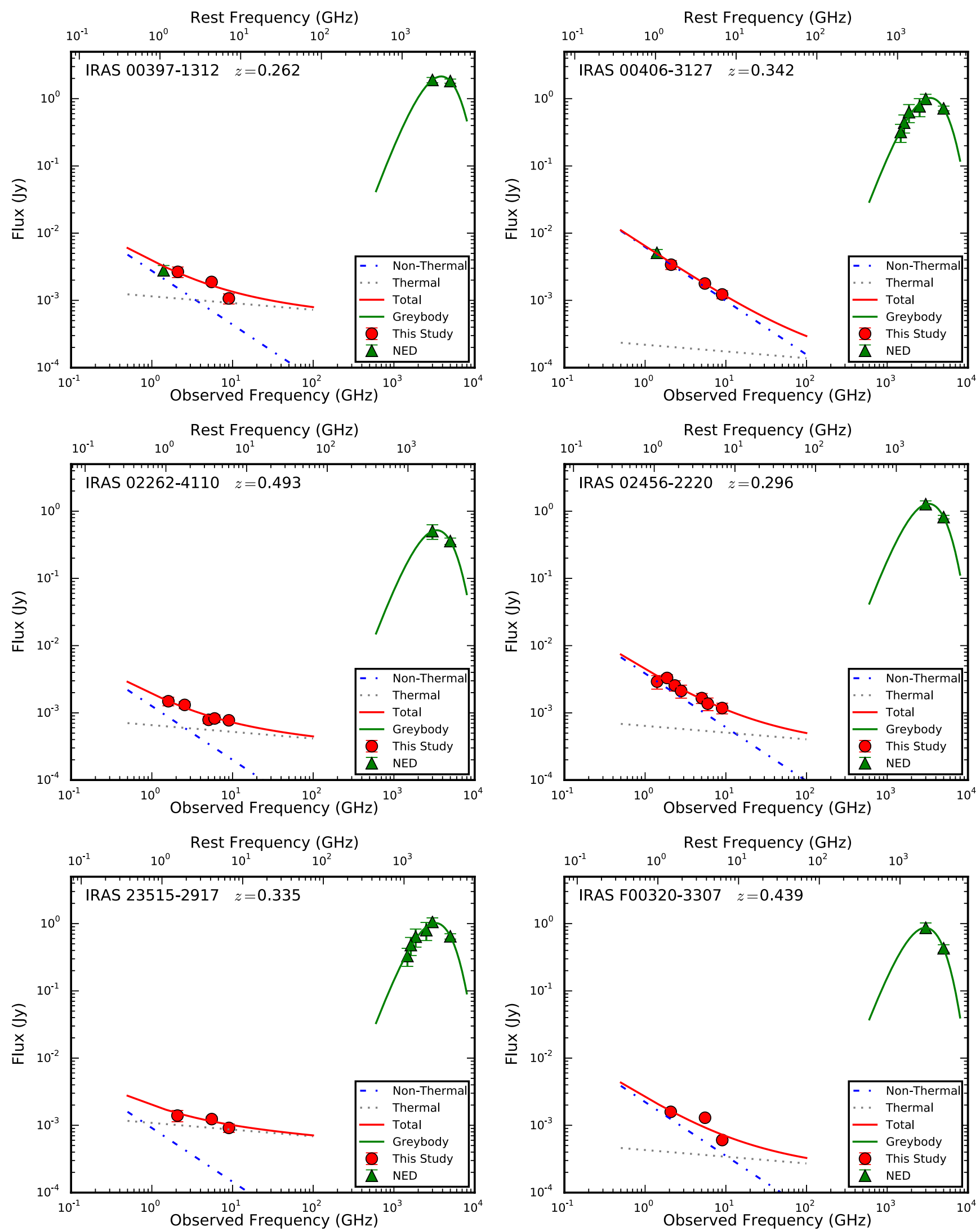

Figure 2. The SEDs of the SFGs from our ULIRG sample. These SEDs are composed of all flux density measurements used during the emission modelling (see Tables A2 and A1), the SFG radio continuum and grey body emission models. 

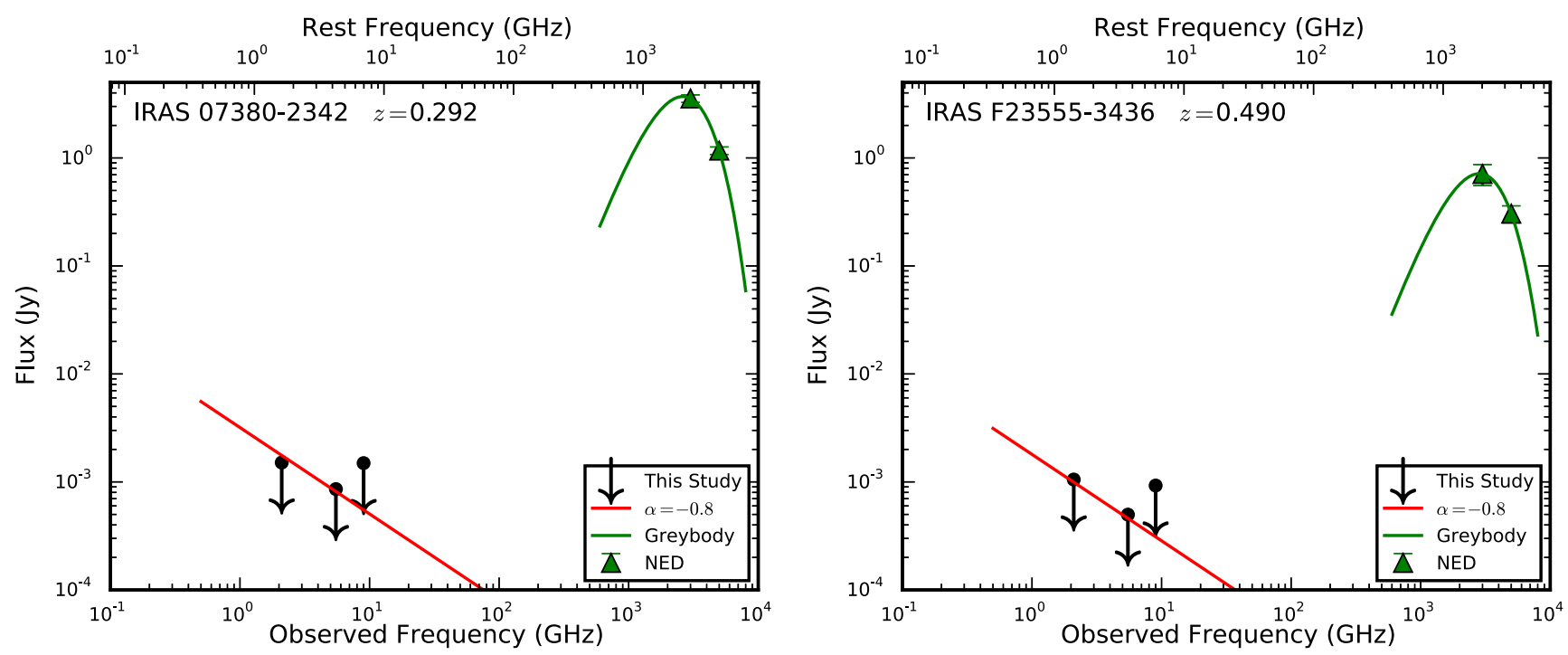

Figure 3. The SEDs of the radio non-detections (consistent with being SFGs) from our ULIRG sample. These SEDs are composed of all flux density measurements used during the emission modelling (see Table A1) and the fitted grey body emission model. The $3 \sigma$ upper limits are presented in sources with non-detections. The solid red line represents the scaled $5.5 \mathrm{GHz} 3 \sigma$ upper limits assuming a spectral index of -0.8 .

Table 2. The best-fitting normalization parameters for the SFG emission model.

\begin{tabular}{lcc}
\hline $\begin{array}{l}\text { Source } \\
\text { IRAS }\end{array}$ & $\begin{array}{c}\text { Non-thermal } \\
\text { normalization } \\
(\mathrm{mJy})\end{array}$ & $\begin{array}{c}\text { Thermal } \\
\text { normalization } \\
(\mathrm{mJy})\end{array}$ \\
\hline F00320-3307 & $2.27 \pm 0.23$ & $0.43 \pm 0.09$ \\
$00397-1312$ & $2.53 \pm 0.80$ & $1.14 \pm 0.30$ \\
$00406-3127$ & $6.00 \pm 0.93$ & $0.21 \pm 0.31$ \\
$02262-4110$ & $1.33 \pm 0.41$ & $0.66 \pm 0.12$ \\
$02456-2220$ & $3.62 \pm 0.79$ & $0.63 \pm 0.35$ \\
$23515-2917$ & $0.88 \pm 0.83$ & $1.08 \pm 0.28$ \\
\hline
\end{tabular}

Table 3. The best-fitting parameters for the grey body emission model. Models were constrained using all available flux density measurements in the range $37<\lambda<500 \mu \mathrm{m}$. The 'fitted temperature' is the $T$ parameter derived during the fitting, whereas the 'rest-frame temperature' has an applied a redshift correction such that $T(1+z)$.

\begin{tabular}{lccc}
\hline $\begin{array}{l}\text { Source } \\
\text { IRAS }\end{array}$ & $\begin{array}{c}60 \mu \mathrm{m} \\
\text { normalization } \\
(\mathrm{Jy})\end{array}$ & $\begin{array}{c}\text { Fitted } \\
\text { temperature } \\
(\mathrm{K})\end{array}$ & $\begin{array}{c}\text { Rest-frame } \\
\text { temperature } \\
(\mathrm{K})\end{array}$ \\
\hline F00320-3307 & $0.76 \pm 0.38$ & $32.1 \pm 2.4$ & $46.2 \pm 3.5$ \\
$00397-1312$ & $0.58 \pm 0.15$ & $41.6 \pm 2.1$ & $52.5 \pm 2.7$ \\
$00406-3127$ & $0.48 \pm 0.11$ & $36.9 \pm 1.5$ & $49.5 \pm 2.0$ \\
$02262-4110$ & $0.25 \pm 0.16$ & $36.6 \pm 3.9$ & $54.7 \pm 5.6$ \\
$02456-2220$ & $0.74 \pm 0.24$ & $35.2 \pm 1.8$ & $45.6 \pm 2.3$ \\
$23515-2917$ & $0.58 \pm 0.13$ & $35.3 \pm 1.4$ & $47.1 \pm 1.9$ \\
\hline
\end{tabular}

fraction is tied to the spectral index. Considering equation (3) in terms of the thermal fraction $\mathrm{TF}$ at $v_{0}$ :

$S_{v}=S_{v_{0}}\left(\mathrm{TF}_{v_{0}}\left(\frac{v}{v_{0}}\right)^{-0.1}+\left(1-\mathrm{TF}_{v_{0}}\right)\left(\frac{v}{v_{0}}\right)^{-0.8}\right)$,

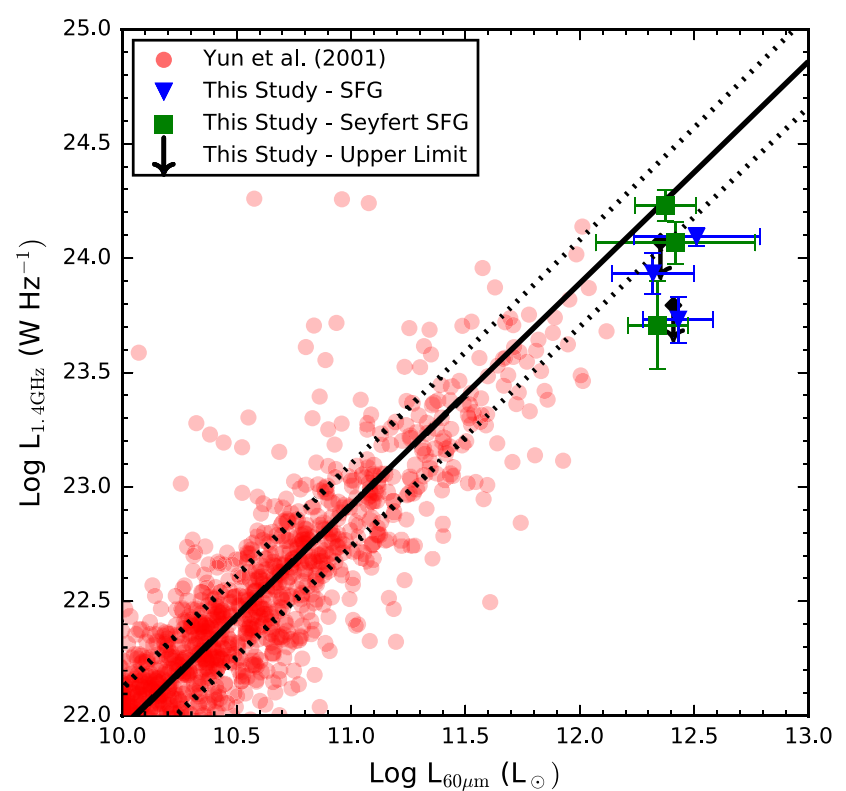

Figure 4. A comparison between the Yun et al. (2001) sample and those in this study. The solid and dotted lines represent a linear best fit and the uncertainty of the normalization component of the Yun et al. (2001) sample. Radio and $60 \mu \mathrm{m}$ luminosities of sources from this study have been $k$-corrected. Galaxies which were not detected in the radio in this project are represented by their $1.4 \mathrm{GHz}$ luminosity upper limits, which have been scaled from their $3 \sigma 5.5 \mathrm{GHz}$ upper limits assuming a spectral index of -0.8 and have been $k$-corrected.

then $\mathrm{TF}_{v_{0}}$ may be expressed in terms of the spectral index $\alpha$ across frequencies $v_{0}$ to $v$ by

$\mathrm{TF}_{\nu_{0}}=\frac{\left(\frac{v}{v_{0}}\right)^{\alpha_{\nu_{0}}^{\nu}}-\left(\frac{\nu}{\nu_{0}}\right)^{-0.8}}{\left(\frac{\nu}{v_{0}}\right)^{-0.1}-\left(\frac{\nu}{v_{0}}\right)^{-0.8}}$. 


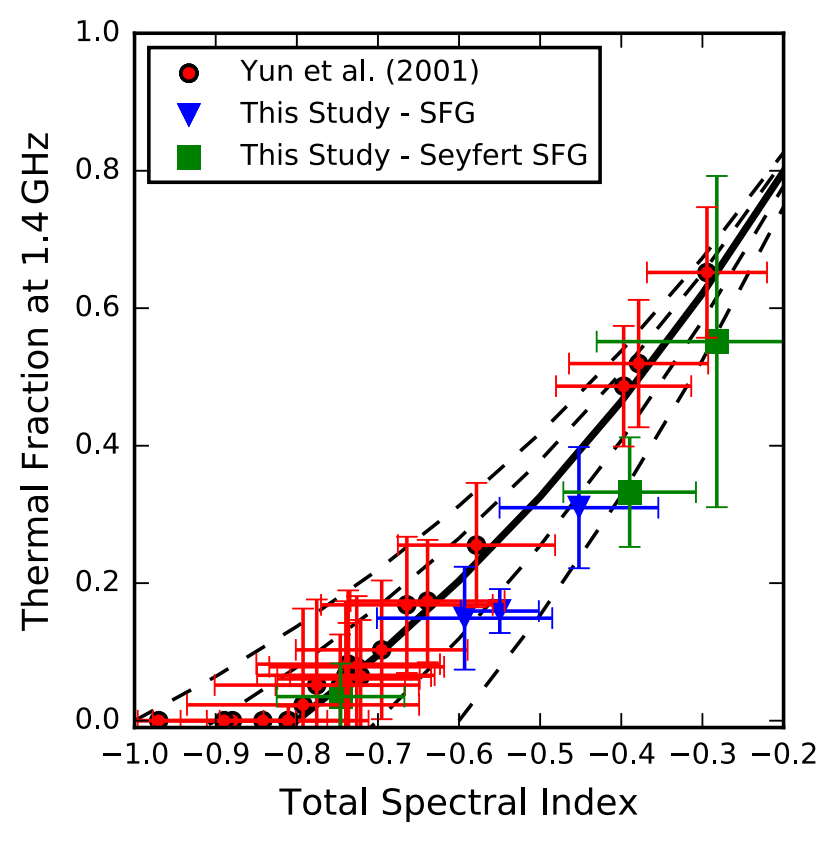

Figure 5. A comparison between the radio spectral index $\left(\alpha_{1.4}^{4.8}\right)$ and the fitted thermal fraction at $1.4 \mathrm{GHz}$ (rest frame) of the SFGs or Seyfert SFGs from this study and 22 sources from Yun et al. (2001). Thermal and nonthermal radio luminosities were calculated in the rest frame at $1.4 \mathrm{GHz}$. The solid line represents the relationship between spectral index and the thermal fraction (see equation 7) over a frequency range of $1.4-4.85 \mathrm{GHz}$. The dashed lines, from left to right, represent how the thermal fraction varies when values of $-1.0,-0.9,-0.7$ and -0.6 are adopted as the spectral index for non-thermal emission.

We include this relationship in Fig. 5, where the spectral index of Yun et al. (2001) sources are calculated over frequency range of $1.4-4.85 \mathrm{GHz}$. Although this relationship is a product of the particular SFG emission model used, it may prove to be a useful diagnostic tool that could allow the spectral index of an object to be used as a proxy when estimating the ratio of thermal to non-thermal emission (as well as potentially other global properties).

In cases where the non-thermal spectral index varies from the adopted -0.8 value, it is straightforward to use equation (7) to model the resulting thermal fraction and how it differs from the case of the -0.8 value used throughout this study. As the spectral index becomes flatter, the thermal fraction becomes more resilient to varying values of non-thermal spectral indices. This effect can be seen in Fig. 5, as the thermal fraction tracks showing the effect of varying the non-thermal spectral index converge as the spectral index increases towards zero.

Both types of radio emission that make up the thermal fraction are caused by different fundamental physical processes with their own time-scales. A high thermal fraction may indicate a young galaxy that has yet to have time to propagate electrons from supernova shocks to emit non-thermal radio emission. Likewise, a low thermal fraction may imply an older galaxy, as the instantaneous thermal emission is less, due to a dwindling SFR, while the ejected relativistic electrons, which produce the non-thermal emission, are still propagating throughout the galaxy.

\subsection{Thermal and non-thermal correlation with FIR}

Using the deconstructed radio continuum emission components from both Yun et al. (2001) and this study we were then able to
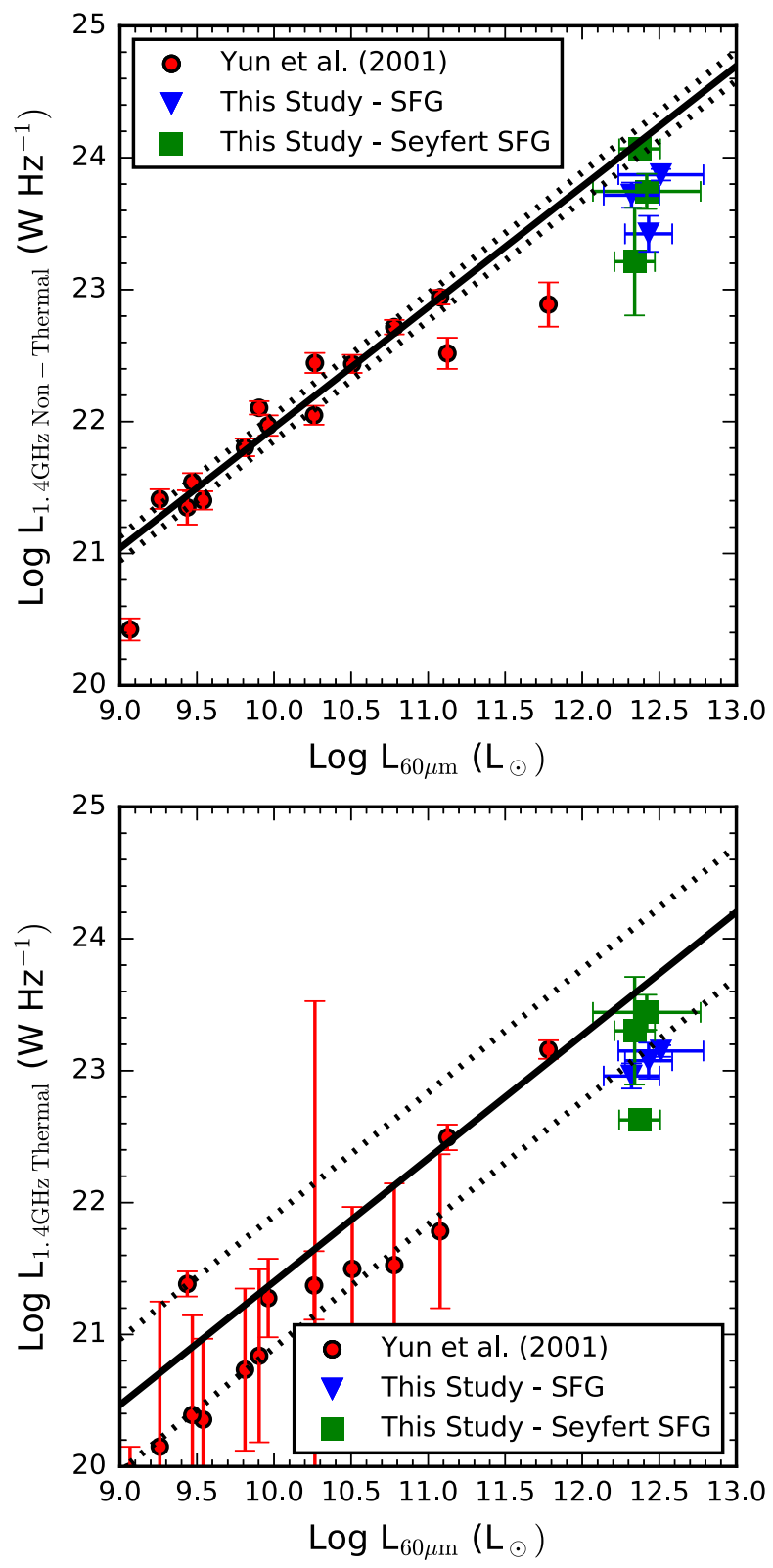

Figure 6. Top: comparison between the $60 \mu \mathrm{m}$ and the fitted non-thermal $1.4 \mathrm{GHz}$ luminosity of 15 sources from Yun et al. (2001) and the six SFG or Seyfert SFG sources from this study. We fit a relation to the 15 Yun et al. (2001) sources, which is presented as a solid line. The dotted lines represent the uncertainty of the fit. Bottom: a comparison between the $60 \mu \mathrm{m}$ and the fitted thermal $1.4 \mathrm{GHz}$ luminosity of 15 sources from Yun et al. (2001) and five SFG sources from this study. The solid and dotted lines represent a fitted relation and the uncertainties of the normalization component fitted to the 15 Yun et al. (2001) sources.

estimate the thermal and non-thermal luminosities at $1.4 \mathrm{GHz}$ for each source. In Fig. 6 we show how these radio luminosities, derived from the fitted emission components of equation (3), correlate with the $60 \mu \mathrm{m}$ luminosities.

These general correlations were obtained from a subset of the Yun et al. (2001) sample derived by applying simple selection criteria. In order to ensure an adequate fit, we excluded sources from Yun et al. (2001) if their $q$ values were less than 1.64 or if the reduced $\chi^{2}$ of the fitted SFG model was greater than 5 . This was done to ensure the 
SFG model was appropriate and was an adequate fit to the available data points. Additionally, if a source had a negligible thermal luminosity $\left(\log L_{1.4 \mathrm{GHz}}^{\mathrm{T}}<19\right)$, then it was also excluded while fitting for both the thermal and non-thermal to infrared luminosity correlations. In total there were 15 of the 22 thermal and non-thermal data points remaining after these criteria were applied to the Yun et al. sample. A bootstrapping method was also used while fitting the correlation as it helped to provide appropriate uncertainties given the relatively small sample size.

The non-thermal radio luminosity at $1.4 \mathrm{GHz}$, shown in Fig. 6 (top), is strongly correlated to the $60 \mu \mathrm{m}$ luminosity. We were able to fit a trend to the 15 Yun et al. (2001) galaxies:

$$
\begin{aligned}
\log \left(L_{1.4 \mathrm{GHz}}^{\mathrm{NT}}\right)= & 0.916[ \pm 0.004] \log \left(L_{60 \mu \mathrm{m}} / \mathrm{L} \odot\right) \\
& +12.80[ \pm 0.04],
\end{aligned}
$$

where $L_{1.4 \mathrm{GHz}}^{\mathrm{NT}}$ is the non-thermal luminosity at $1.4 \mathrm{GHz}$. These parameters were constrained using the fitted results of 10000 randomly selected subsamples of the 15 sources (with repetition allowed).

At $L_{60 \mu \mathrm{m}}<10^{11} \mathrm{~L}_{\odot}$ this correlation is especially strong, with little scatter from the best-fitting correlation. Towards the upper end of the fitted correlation there appears to be a slight increase in the scatter with two sources roughly 0.2 dex below the main trend. This increased scatter is also seen in the SFG sources from this study (although possibly subject to our object selection method discussed in Section 6).

Likewise, the thermal radio luminosity at $1.4 \mathrm{GHz}$ is also shown to be correlated with $60 \mu \mathrm{m}$ luminosities in Fig. 6 (bottom). We fit a correlation to the Yun et al. (2001) luminosity estimates using the bootstrapping method outlined above:

$$
\begin{aligned}
\log \left(L_{1.4 \mathrm{GHz}}^{\mathrm{T}}\right)= & 0.93[ \pm 0.05] \log \left(L_{60 \mu \mathrm{m}} / \mathrm{L}_{\odot}\right) \\
& +12.10[ \pm 0.51]
\end{aligned}
$$

where $L_{1.4 \mathrm{GHz}}^{\mathrm{T}}$ is the thermal luminosity at $1.4 \mathrm{GHz}$. The uncertainties of the fitted components, especially the normalization component, are higher when compared to the fitted non-thermal trend.

\subsection{Thermal fraction and $q$}

We compared the estimated thermal fraction at $1.4 \mathrm{GHz}$ obtained from the fitted SFG models to the $q$ parameter (Fig. 7) for sources from this study and Yun et al. (2001). Sources whose fitted spectral index was outside the range $-0.8<\alpha<-0.1$ were excluded from Fig. 7, as thermal fractions of sources whose values are outside this range cannot be represented by the SFG model in its current form (returned as either 100 or 0 per cent, as demonstrated in Fig. 5).

We see in Fig. 7 the mean $q$ value of Yun et al. (2001) (represented as a dashed line) appears to mark a transition from low thermal fractions to increased values accompanied by larger intrinsic scatter. For the 18 sources where $q<2.34$ we find the mean and standard deviation (SD) of the thermal fraction to be 0.08 and 0.12 , respectively. Of the eight sources where $q>2.34$, the mean and $\mathrm{SD}$ of their thermal fraction corresponds to 0.38 and 0.18 . A two sided Kolmogorov-Smirnov test with each of these groups returns a $p$-value below 1 percent, indicating that there are two different underlying distributions present.

Marvil et al. (2015) show that the $q$ parameter may be related to the thermal fraction of a source. Using SFR conversion factors based on radio thermal emission originating from resolved $\mathrm{H}$ II regions and IR (see Kennicutt 1998; Murphy et al. 2011), then the radio thermal emission component may be described in terms of the total FIR

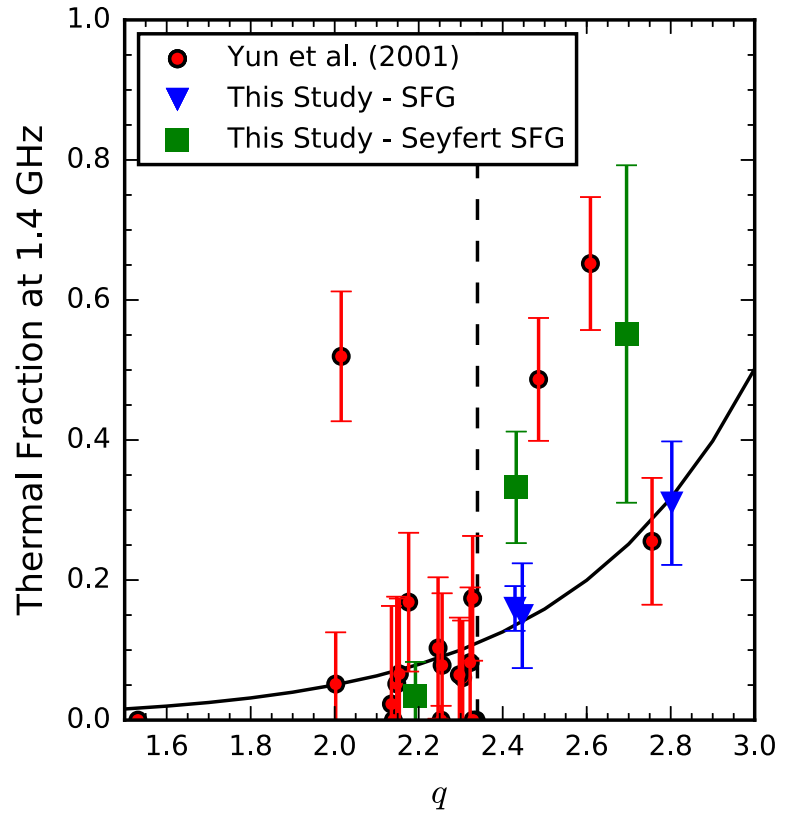

Figure 7. A comparison between the $q$ parameter against the fitted thermal fraction at $1.4 \mathrm{GHz}$ (rest frame) of the six detected SFGs or Seyfert SFGs from this study and 20 sources from Yun et al. (2001). The dashed vertical line represents the mean $q$ value of Yun et al. (2001) $(q=2.34)$. The solid line is equation (8) from Marvil, Owen \& Eilek (2015) and relates the $q$ parameter to a thermal fraction.

emission. Since the $q$ parameter and this derived expression share this common FIR term, the thermal fraction can be expressed in terms of $q$. The solid line shown in Fig. 7 represents this relationship. Sources with small values of $q$ which would classically be described as radio-excess, could alternatively be interpreted as having low thermal fractions using this relation (Marvil et al. 2015).

\section{DISCUSSION}

We have constrained the multiple radio emission components that make up a typical luminous SFG (at higher redshifts and luminosities). This type of modelling shows that there exists a measurable thermal emission component that contributes to the radio continuum at $v<10 \mathrm{GHz}$. Although the uncertainties associated with the thermal components are high for three of the six fitted SFG models, this can be attributed to the frequency range of flux density measurements used in the radio continuum and low thermal fractions. Typically, the thermal component of an SFG does not become a significant fraction of the radio continuum until frequencies above $10 \mathrm{GHz}$. We find it encouraging that despite a lack of measurements in this regime that we were still able to estimate this mechanism. Once the $10-100 \mathrm{GHz}$ frequency range is sampled, a more robust SFG model could be constrained.

\subsection{Object selection}

ULIRGs were specifically targeted for inclusion in this study for two reasons. The first is that they are a useful representative sample of the types of active galaxies (in terms of star formation and luminosities) which are expected to be detected at higher redshifts with the next generation of radio telescopes, including ASKAP, MeerKAT, JVLA, LOFAR, eMERLIN and SKA. Secondly, the strong emission seen 
in the infrared is indicative of high SFR. Assuming that the nonthermal emission is a delayed tracer of star formation, we expect that the thermal component would be a significant fraction of the radio continuum at $1.4 \mathrm{GHz}$, allowing it to be more easily disentangled from the overall spectrum. This property is especially applicable to high-redshift objects, where the observed $1.4 \mathrm{GHz}$ radio flux density will include a larger fraction of thermal emission than observed in local galaxies. Given that of the six SFG galaxies identified here, five were fitted with a measurable thermal component, this seems to be a valid approach.

A side effect of our selection criteria is, as mentioned in Section 5.2, an apparent deficit in the radio luminosity of the six SFG objects from this study, given the fitted FRC. A source must have a FIR luminosity in excess of $10^{12.5} \mathrm{~L}_{\odot}$ to be included in our sample. Though this was successful in identifying galaxies with high SFR and a measurable thermal component, it also had the effect of selecting sources with a higher mean $q$ value once AGN contamination was corrected, thereby producing the radio luminosity deficiency observed.

To confirm the origin of our higher $q$ values, we obtained the RIFSCz (Wang et al. 2014) and applied the same selection criteria as described in Section 2.1, with the exception of replacing item 3 $\left(L_{\mathrm{FIR}}>10^{12.5} \mathrm{~L}_{\odot}\right)$ with the requirement for a source to have a $60 \mu \mathrm{m}$ flux $>0.5 \mathrm{Jy}$. This amended criteria targeted the region between the upper limit of the Yun et al. (2001) sample and our own. After removing all sources without a listed $1.4 \mathrm{GHz}$ flux density, there were 26 objects whose properties matched the amended selection criterion (including sources IRAS F00397-1312 and IRAS F00406-3127 from this study). We find that this sample is equally scattered around the FRC from Yun et al. (2001), extrapolated to higher luminosities.

\subsection{Thermal fraction}

One of the properties of the fitted SFG emission model is that the thermal fraction is tied closely to the spectral index of a galaxy (Fig. 5). This relationship allows an estimate of the thermal fraction, obtained from the spectral index, to be used without the need for detailed SED modelling. For instance, this thermal fraction may be used as a way to indicate the starburst age of a particular galaxy. The thermal emission, caused by free-free-type interactions between particles in ionized clouds of hydrogen surrounding HMS, is an instantaneous emission characterized by a relatively flat spectrum. On the other hand, the steep spectrum of the synchrotron emission is delayed due to the lifespan of OB stars $\left(\sim 10^{7} \mathrm{yr}\right)$ and the time required for relativistic electrons, ejected from the shocks of supernovae, to diffuse $\left(\sim 10^{4} \mathrm{yr}\right)$.

We show in Fig. 7 that sources with higher values of $q(q>$ $2.34)$ tend to have a larger thermal fraction. Additionally the thermal fraction of sources in this region have a larger scatter than seen in the radio sources with lower values of $q(q<2.34)$. Potentially, this may be explained by the time-scales associated with each emission mechanism, as well as the duration of the starburst itself. A younger starburst will have a high thermal fraction and a high value of $q$ as the non-thermal emission is delayed and absent from its radio continuum. As the starburst ages, the $q$ parameter and thermal fraction will decrease, due to the time-delayed, non-thermal radio emission increasing, and also the declining SFR reducing the amount of instantaneous thermal emission.

The scatter of the thermal fraction when compared against $q$ could be influenced by the duration of the starburst. For instance if star formation ceases before the non-thermal emission commences, then the $q$ parameter will possibly increase if the IR emission persists longer than the thermal emission, but then decreases once the IR emission begins to decline. The thermal fraction will decline only very slowly if the non-thermal component is low. However, once the non-thermal component commences the thermal fraction will decline quickly and $q$ will decrease almost as rapidly. Alternatively, if the non-thermal emission begins to contribute to the radio continuum while star formation is still ongoing, then the thermal fraction and $q$ will decrease more gradually.

\subsection{Correlation of decomposed radio luminosities}

Through the application of the SFG emission model we were able to estimate thermal and non-thermal luminosities in a collection of 28 sources across the two studies. These decomposed components themselves correlate with $60 \mu \mathrm{m}$ luminosity across many orders of magnitudes in a manner similar to that of the traditional FRC. Although these trends are clearly present, the thermal to $60 \mu \mathrm{m}$ luminosity correlation is less constrained. Given that the 22 sources from Yun et al. (2001) had their SFG model constrained with only three measurements across a frequency range dominated by non-thermal emission, the thermal component of the resulting fit will inherently carry with it a higher uncertainty. Consequently, the resulting fitted trend is less well constrained as the non-thermal correlation.

During the fitting of these correlations, we elected to fit only to sources from the Yun et al. (2001) sample and not to include the SFG sources from this study. This was done because, as outlined above, our sample of SFG-type objects trend towards high $q$ (as can be seen in Fig. 4) and would have diminished the effectiveness of the fitted correlations when using the decomposed radio luminosities. As previously discussed, sources that were selected for inclusion in this study were based on their high total FIR luminosities, as it is likely this excess infrared emission originates from a high rate of star formation and possibly a younger stellar age. Although this approach helped to identify sources where we were able to constrain the thermal radio continuum properties of our sample, it has resulted in sources with higher $q$.

\subsection{Radio-weak AGN contribution}

We have attempted to remove AGN of varies classes from our sample by examining their $q$ parameter, IRAS flux density ratios and IR template fitting results from Wang et al. (2014). Although we are confident that the IR of our sample is powered by intense star formation, we are unable to completely dismiss the possibility of a radio-weak AGN contributing to the observed radio continuum. To investigate how the thermal fraction and $q$ parameter would vary in such situations we have performed simulations on each of our sources by subtracting an assumed AGN contribution from our radio continuum measurements and re-fitting the SFG model using the remaining flux. We defined the AGN spectra as a simple power law (i.e. $S_{v}=N v^{\alpha}$ ), whose parameter space was defined as $-1.3<\alpha$ $<-0.1$ (a typical range for AGN) and $0<N<0.2(A+B)$ (i.e. up to 20 per cent of the rest frame $1.4 \mathrm{GHz}$ ), where $A$ and $B$ are normalizations components of the baseline SFG model (Table 2) for some source.

In Fig. 8 we show how the thermal fraction and $q$ parameter vary after subtracting an assumed AGN spectra from the radio continuum. We see that when a steep spectrum is assumed the thermal fraction will increase as a portion of the non-thermal emission, with a $\alpha=-0.8$, is subtracted from observed radio continuum. Likewise, when a flat spectrum is subtracted the thermal fraction will 

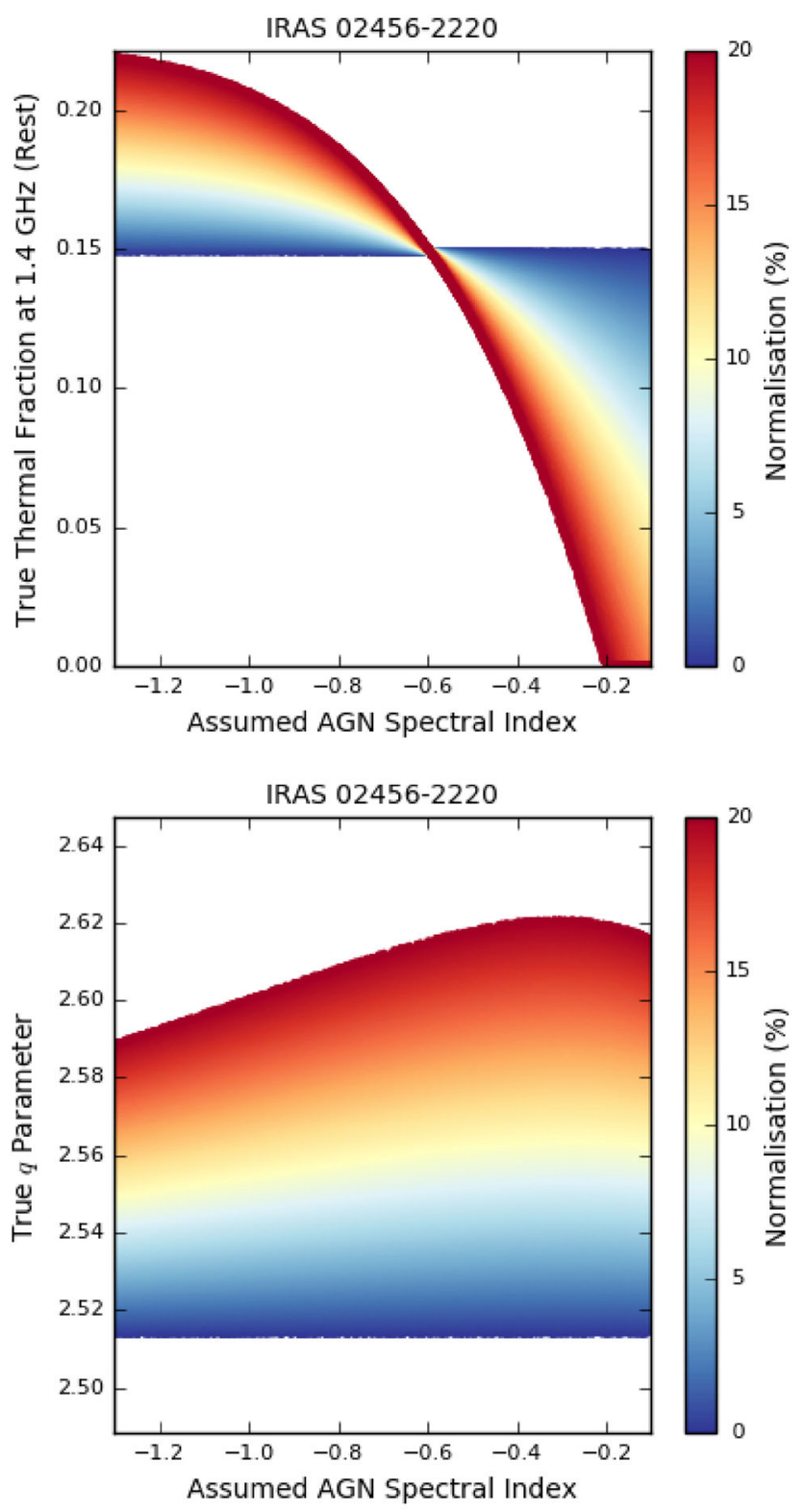

Figure 8. A plot showing how the thermal fraction (top) and $q$ parameter (bottom) vary after removing a simulated AGN (described as a simple power law) from the radio continuum of IRAS 02456-2220 and refitting the SFG model (equation 3). The colour indicates the normalization of the AGN component, which is taken as a fraction of the $S_{\nu_{0}}$ of the baseline fitted SFG model.

decrease as a portion of the thermal component is removed. The point of inflection (when the thermal fraction begins to decrease with increasing AGN spectral index) occurs at an assumed AGN spectral index of $\alpha=-0.6$, which corresponds to the actual spectral index of IRAS 02456-2220. Throughout the simulations the $q$ parameter reacts fairly consistently across a range of assumed AGN spectral indices, and is more affected by the normalization component. The values of $q$ remaining within $1 \sigma$ of the mean of the Yun et al. (2001) sample (Fig. 1).

These simulations suggest that if a radio-weak AGN contribution were present its effects would be small. However, there is no evidence to suggest that such a radio-weak AGN contribution exists for any of our sources.

\section{CONCLUSIONS}

With our ATCA data we have measured the intrinsic thermal and non-thermal emission that comprise the radio continuum. The ULIRGs included as part of this study, with their high infrared luminosities and vigorous star formation, are an excellent representative sample of the class of galaxies which are observed in current and future of deep, extragalactic radio surveys. Understanding the physical contribution of star formation process to the radio regime is necessary to characterize these distant galaxies. To this end, we have shown through our emission modelling the following.

(i) The intrinsic thermal and non-thermal emission components that make up the radio continuum can be separated with as few as three flux density measurements. The steep spectrum of nonthermal emission is easily distinguishable from the relatively flat spectrum of thermal emission.

(ii) Both the thermal and non-thermal emission components form their own well-defined correlations with the $60 \mu \mathrm{m}$ luminosities. Like the FRC, these correlations exist over many orders of magnitude with relatively small scatter.

(iii) The thermal fraction of a fitted SFG model is tied closely to the fitted spectral index of the object. Given the wide bandwidth filters now fitted on the ATCA and the Karl G. Janksy Very Large Array (JVLA), this will be very useful in obtaining initial estimates of the thermal emission of SFGs without having to fit a complete SFG model to its radio continuum.

(iv) With increasing values of $q$ there is an increase in the value and scatter of the thermal fraction. This could be due to the differing time-scales associated with thermal and non-thermal radio emission originating from high-mass star formation. Galaxies with younger starbursts may have a higher $q$ and thermal fraction due to the delayed non-thermal emission mechanism. Likewise, galaxies with older starbursts may have a lower $q$ and thermal fractions due to declining SFRs (thereby reducing the amount of instantaneous thermal emission) and increasing non-thermal emission from earlier bursts of star formation.

\section{ACKNOWLEDGEMENTS}

We would like to thank the referee, J. Condon, for his extensive and constructive feedback which greatly improved the paper. The Australia Telescope Compact Array is part of the Australia Telescope National Facility which is funded by the Commonwealth of Australia for operation as a National Facility managed by CSIRO. This paper includes archived data obtained through the Australia Telescope Online Archive (http://atoa.atnf.csiro.au). NS is the recipient of an ARC Future Fellowship. This research has made use of the NASA/IPAC Extragalactic Database (NED) which is operated by the Jet Propulsion Laboratory, California Institute of Technology, under contract with the National Aeronautics and Space Administration. Special thanks to E. J. Crawford, R. Braun and D. Farrah for their assistance throughout the project.

\section{REFERENCES}

Allen D. A., Norris R. P., Meadows V. S., Roche P. F., 1991, MNRAS, 248, 528

Appleton P. N. et al., 2004, ApJS, 154, 147

Bell E. F., 2003, ApJ, 586, 794 
Bevington P. R., 1969, Data Reduction and Error Analysis for the Physical Sciences. McGraw-Hill, New York

Braun R., Popping A., Brooks K., Combes F., 2011, MNRAS, 416, 2600

Bressan A., Silva L., Granato G. L., 2002, A\&A, 392, 377

Clemens M. S., Vega O., Bressan A., Granato G. L., Silva L., Panuzzo P., 2008, A\&A, 477, 95

Condon J. J., 1992, ARA\&A, 30, 575

Condon J. J., Cotton W. D., Greisen E. W., Yin Q. F., Perley R. A., Taylor G. B., Broderick J. J., 1998, AJ, 115, 1693

da Cunha E. et al., 2015, ApJ, 806, 110

de Jong T., Klein U., Wielebinski R., Wunderlich E., 1985, A\&A, 147, L6

Dressel L. L., Condon J. J., 1978, ApJS, 36, 53

Garrett M. A., 2002, A\&A, 384, L19

Gooch R., 2011, Karma: Visualisation Test-Bed Toolkit. Astrophysics Source Code Library

Gregory P. C., Scott W. K., Douglas K., Condon J. J., 1996, ApJS, 103,427

Hambly N. C. et al., 2001, MNRAS, 326, 1279

Han Y., Han Z., 2012, ApJ, 749, 123

Helou G., Walker D. W., eds 1988, Infrared Astronomical Satellite (IRAS) Catalogs and Atlases. Vol. 7, The Small Scale Structure Catalog. NASA, Washington, DC

Helou G., Soifer B. T., Rowan-Robinson M., 1985, ApJ, 298, L7

Hughes A., Wong T., Ekers R., Staveley-Smith L., Filipovic M., Maddison S., Fukui Y., Mizuno N., 2006, MNRAS, 370, 363

Ivison R. J. et al., 2010, A\&A, 518, L31

Kennicutt R. C., Jr, 1998, ARA\&A, 36, 189

Klaas U. et al., 2001, A\&A, 379, 823

Kovács A., Chapman S. C., Dowell C. D., Blain A. W., Ivison R. J., Smail I., Phillips T. G., 2006, ApJ, 650, 592

Lisenfeld U., Voelk H. J., Xu C., 1996, A\&A, 314, 745

Mao M. Y., Norris R. P., Emonts B., Sharp R., Feain I., Chow K., Lenc E., Stevens J., 2014, MNRAS, 440, L31

Marvil J., Owen F., Eilek J., 2015, AJ, 149, 32

Moshir M., 1990, in IRAS Faint Source Catalogue, version 2.0

Murphy E. J., 2009, ApJ, 706, 482

Murphy E. J. et al., 2006, ApJ, 651, L111

Murphy E. J. et al., 2011, ApJ, 737, 67

Murphy E. J. et al., 2014, preprint (arXiv:1412.5677)

Nardini E., Risaliti G., Watabe Y., Salvati M., Sani E., 2010, MNRAS, 405, 2505

Norris R. P., Lenc E., Roy A. L., Spoon H., 2012, MNRAS, 422, 1453

Price R., Duric N., 1992, ApJ, 401, 81

Rouan D., 2011, in Gargaud M., Amils R., Quintanilla J., Cleaves Henderson -James (Jim) I., Irvine W., Pinti D., Viso M., eds, Encyclopedia of Astrobiology. Springer-Verlag, Berlin

Rowan-Robinson M., 2000, MNRAS, 316, 885

Roy A. L., Norris R. P., Kesteven M. J., Troup E. R., Reynolds J. E., 1998, MNRAS, 301, 1019

Sault R. J., Wieringa M. H., 1994, A\&AS, 108, 585

Sault R. J., Teuben P. J., Wright M. C. H., 2011, MIRIAD: Multi-channel Image Reconstruction, Image Analysis, and Display. Astrophysics Source Code Library, record ascl:1106.007

Seymour N. et al., 2008, MNRAS, 386, 1695

Seymour N., Huynh M., Dwelly T., Symeonidis M., Hopkins A., McHardy I. M., Page M. J., Rieke G., 2009, MNRAS, 398, 1573

Seymour N. et al., 2011, MNRAS, 413, 1777

Spoon H. W. W., Armus L., Marshall J. A., Bernard-Salas J., Farrah D., Charmandaris V., Kent B. R., 2009, ApJ, 693, 1223

Symeonidis M. et al., 2013, MNRAS, 431, 2317

van der Kruit P. C., 1971, A\&A, 15, 110

van der Kruit P. C., 1973, A\&A, 29, 263

Véron-Cetty M.-P., Véron P., 2006, A\&A, 455, 773

Vlahakis C., Eales S., Dunne L., 2007, MNRAS, 379, 1042

Voelk H. J., 1989, A\&A, 218, 67

Wang L., Rowan-Robinson M., Norberg P., Heinis S., Han J., 2014, MNRAS, 442, 2739
Wilson W. E. et al., 2011, MNRAS, 416, 832

Yun M. S., Carilli C. L., 2002, ApJ, 568, 88

Yun M. S., Reddy N. A., Condon J. J., 2001, ApJ, 554, 803

\section{APPENDIX A: FLUX DENSITY MEASUREMENTS}

Table A1. All additional flux density measurements collected from NED for each of the sources observed as a part of this study. For a measurement to be included it was required to be a broad-band measurement in the ranges $0.1<v<100 \mathrm{GHz}$ or $37<\lambda<500 \mu \mathrm{m}$. References are Helou \& Walker (1988, HW88); Moshir (1990, M90); Condon et al. (1998, C98); Klaas et al. (2001, K01); Spoon et al. (2009, S09).

\begin{tabular}{|c|c|c|c|c|}
\hline $\begin{array}{l}\text { Source name } \\
\text { IRAS }\end{array}$ & $\begin{array}{c}\text { Frequency } \\
\quad(\mathrm{GHz})\end{array}$ & $\begin{array}{l}\text { Flux density } \\
\qquad(\mathrm{mJy})\end{array}$ & Telescope & Reference \\
\hline \multirow{4}{*}{ F00183-7111 } & 1920.00 & $540.00 \pm 80.00$ & MIPS & S09 \\
\hline & 3000.00 & $1190.00 \pm 119.00$ & IRAS & M90 \\
\hline & 4200.00 & $1500.00 \pm 225.00$ & MIPS & S09 \\
\hline & 5000.00 & $1200.00 \pm 83.70$ & IRAS & M90 \\
\hline \multirow{2}{*}{ F00320-3307 } & 3000 & $869 \pm 156$ & IRAS & M90 \\
\hline & 5000 & $430 \pm 60$ & $I R A S$ & M90 \\
\hline \multirow{3}{*}{ 00397-1312 } & 1.40 & $2.80 \pm 0.50$ & VLA & C98 \\
\hline & 3000 & $1900 \pm 170$ & IRAS & M90 \\
\hline & 5000 & $1830 \pm 130$ & IRAS & M90 \\
\hline \multirow{7}{*}{$00406-3127$} & 1.40 & $5.1 \pm 0.6$ & VLA & C98 \\
\hline & 1470 & $320 \pm 100$ & ISO & K01 \\
\hline & 1620 & $440 \pm 130$ & ISO & K01 \\
\hline & 1860 & $630 \pm 190$ & ISO & K01 \\
\hline & 2520 & $770 \pm 230$ & ISO & K01 \\
\hline & 3000 & $994 \pm 169$ & IRAS & M90 \\
\hline & 5000 & $718 \pm 57$ & IRAS & M90 \\
\hline \multirow{2}{*}{$02262-4110$} & 3000 & $517 \pm 124$ & IRAS & M90 \\
\hline & 5000 & $390 \pm 40$ & IRAS & M90 \\
\hline \multirow{2}{*}{$02456-2220$} & 3000 & $1270 \pm 150$ & $I R A S$ & M90 \\
\hline & 5000 & $816 \pm 49$ & $I R A S$ & M90 \\
\hline \multirow{6}{*}{$03538-6432$} & 1470.00 & $620.00 \pm 186.00$ & ISO & K01 \\
\hline & 1620.00 & $850.00 \pm 255.00$ & ISO & K01 \\
\hline & 1860.00 & $1070.00 \pm 321.00$ & ISO & K01 \\
\hline & 2520.00 & $1370.00 \pm 411.00$ & ISO & K01 \\
\hline & 3000.00 & $1300.00 \pm 195.00$ & IRAS & M90 \\
\hline & 5000.00 & $988.00 \pm 49.40$ & IRAS & M90 \\
\hline \multirow{2}{*}{ F04565-2615 } & 3000.00 & $577.00 \pm 133.00$ & IRAS & M90 \\
\hline & 5000.00 & $296.00 \pm 32.60$ & IRAS & M90 \\
\hline \multirow{2}{*}{$07380-2342$} & 3000 & $3550 \pm 280$ & IRAS & HW88 \\
\hline & 5000 & $1170 \pm 90$ & IRAS & HW88 \\
\hline \multirow{2}{*}{ F23529-2119 } & 3000.00 & $627.00 \pm 132.00$ & IRAS & M90 \\
\hline & 5000.00 & $327.00 \pm 55.50$ & IRAS & M90 \\
\hline \multirow{6}{*}{$23515-2917$} & 1470 & $330 \pm 100$ & ISO & K01 \\
\hline & 1620 & $480 \pm 140$ & ISO & K01 \\
\hline & 1860 & $640 \pm 190$ & ISO & K01 \\
\hline & 2520 & $800 \pm 240$ & ISO & K01 \\
\hline & 3000 & $1060 \pm 160$ & IRAS & M90 \\
\hline & 5000 & $648 \pm 58$ & IRAS & M90 \\
\hline \multirow{2}{*}{ F23555-3436 } & 3000 & $711 \pm 157$ & IRAS & M90 \\
\hline & 5000 & $305 \pm 54$ & IRAS & M90 \\
\hline
\end{tabular}




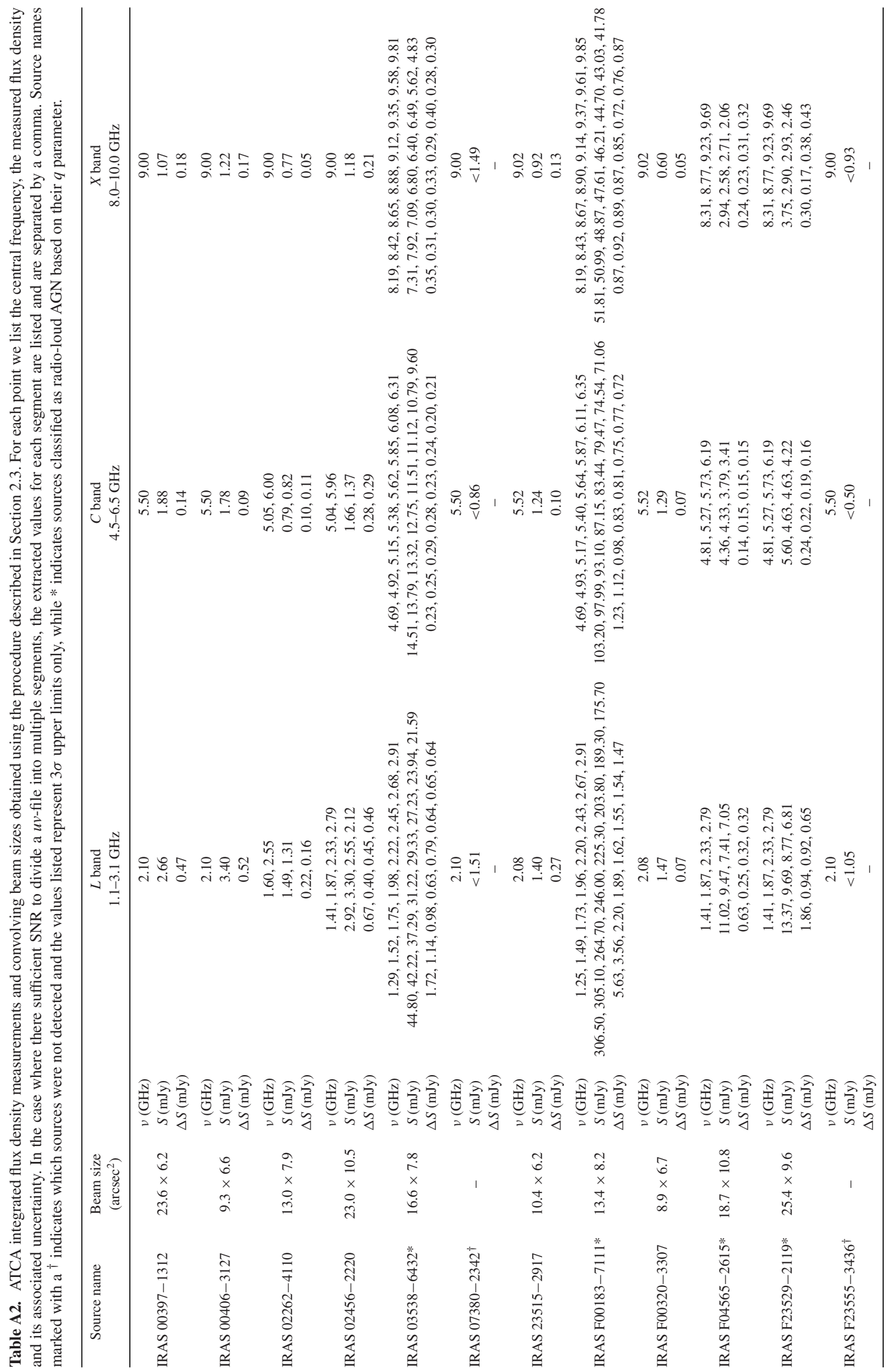




\section{APPENDIX B: SOURCES IN THE LITERATURE}

Here we briefly outline the literature available for the SFG and radio non-detection sources that remain in our sample.

IRAS F00320-3307. There exists no classification of this source in the literature. We classify it as SFG based on its location on the FRC.

IRAS 00397-1312. A compact starburst with clear polycyclic aromatic hydrocarbon (PAH) detections from the Spitzer spectrograph (Nardini et al. 2010).

IRAS 00406-3127. Classified as a Seyfert 2 type galaxy (Nardini et al. 2010). Roy et al. (1998) did not detect its radio AGN core using a $275 \mathrm{~km}$ array between the 64-m Parkes and 70-m Tidbinbilla antennas. Therefore we assume that there is no significant radio AGN component in the observed radio continuum.

IRAS 02262-4110. Véron-Cetty \& Véron (2006) classified this object as a Seyfert 1 type galaxy based on the ratio of the $\mathrm{H} \beta /[\mathrm{O} \mathrm{III}] \lambda 5007$ integrated line fluxes. However given its soft $\mathrm{X}$ ray luminosity, which is $\log \left(L_{x}\right)=45.1 \mathrm{erg} \mathrm{s}^{-1}$, and its power law exhibited from X-ray to FIR, this source could potentially be a broad line QSO. As its $q$ parameter shows no excess emission, we assume that there is no significant AGN component observed in the radio continuum. Likewise, the template fitting performed by Wang et al. (2014) suggests no significant AGN component in the FIR.
IRAS 02456-2220. There exists no classification of this source in the literature. We classify it as SFG based on its location on the FRC.

IRAS 07380-2342. This is one of the radio non-detected sources in our sample. Rowan-Robinson (2000) classified this object as a narrow-line AGN.

IRAS 23515-2917. A Seyfert 2 type galaxy (Nardini et al. 2010) that is possibly interacting with a nearby object (Allen et al. 1991). This potential merger may have triggered a recent burst of star formation, causing the high luminosity seen in the radio and FIR. Its flat radio spectrum is currently being modelled as a starburst in which the optically thin free-free emission dominates across all frequencies. An alternative interpretation is this flattening could be a result of the non-thermal component being suppressed by free-free absorption effects at $L$ band, thereby producing a higher thermal fraction. Comprehensive modelling would be needed with more complete radio continuum data in order to investigate this alternate interpretation further.

IRAS F23555-3436. This is one of the radio non-detected sources in our sample and there exists no classification of this source in the literature.

This paper has been typeset from a $\mathrm{TE}_{\mathrm{E}} \mathrm{X} / \mathrm{A} \mathrm{T} \mathrm{E}$ file prepared by the author. 